Voix et Images

volxetimages

\title{
Converser à Scoudouc ou ailleurs. Installations poétiques et espaces dialogiques
} Conversing in Scoudouc or Elsewhere. Poetic Installations and Dialogic Spaces

\section{Conversar en Scoudouc o en otra parte. Instalaciones poéticas y espacios dialógicos}

\section{Thierry Bissonnette}

Volume 35, numéro 1 (103), automne 2009

Herménégilde Chiasson

URI : https://id.erudit.org/iderudit/038568ar

DOI : https://doi.org/10.7202/038568ar

Aller au sommaire du numéro

Éditeur(s)

Université du Québec à Montréal

ISSN

0318-9201 (imprimé)

1705-933X (numérique)

Découvrir la revue

\section{Citer cet article}

Bissonnette, T. (2009). Converser à Scoudouc ou ailleurs. Installations poétiques et espaces dialogiques. Voix et Images, 35(1), 29-36.

https://doi.org/10.7202/038568ar
Résumé de l'article

Dans une grande partie de son oeuvre écrite, Herménégilde Chiasson manifeste un attrait profond pour l'art contemporain, notamment pour le travail sur le contexte effectué par ce dernier. Cela l'amène à maintes reprises à s'inspirer de la pratique de l'installation pour composer ses recueils que j'analyserai en faisant ressortir le lien entre cette pratique composite et la valorisation du travail du lecteur, laquelle fait de l'oeuvre du poète une véritable conversation. 


\title{
CONVERSER À SCOUDOUC OU AILLEURS. \\ INSTA LLATIONS POÉTIQUES \\ ET ESPACES DIALOGIQUES
}

$+++$

\author{
THIERRY BISSONNETTE \\ Université Laurentienne
}

Pour le praticien de l'installation ${ }^{1}$, le contexte spatiotemporel et les circonstances interprétatives font généralement partie de l'œuvre proposée, laquelle trouve d'ailleurs là un ressort distinctif de son dynamisme. Fabriquer une installation implique une perspective sur le lieu de l'œuvre, de même qu'une anticipation du comportement du spectateur, dont les attentes sont incluses dans un dialogue ludique et souvent autoréférentiel (dialogue sur le dialogue, monstration de ce dernier) qui s'oppose à la clôture monologique de l'objet. Particulièrement développée au cours des années 1960, l'installation combine différents genres artistiques et incite à une réflexion sur la nature de l'art en introduisant parfois des éléments a priori non esthétiques dans le contexte de la galerie d'art (pensons à l'emblématique urinoir de Marcel Duchamp), voire en sortant l'art hors des galeries (créations in situ), croisant les registres de manière à impliquer le spectateur dans l'élaboration et la définition de l'œuvre.

Outre sa pratique professionnelle de l'art pictural, Herménégilde Chiasson introduit fréquemment dans sa poésie une dimension «installative» en y transposant virtuellement le type d'interactions observable en galerie. J'analyserai ici cette transposition indépendamment de la production visuelle de l'écrivain en décrivant chez Chiasson une poétique de l'« objet trouvé», pour ensuite considérer de plus près le rôle actif du récepteur qu'engagent plusieurs de ses livres récents.

$$
+++
$$

1 Sur cette forme importante de l'art contemporain et ses assises théoriques, voir notamment Daniel Buren, «Fonction du musée», Les écrits (1965-1990), tome 1, 1965-1976, Bordeaux, CAPC/Musée d'art contemporain de Bordeaux, 2000, p. 169-173; Brian O'Doherty, Inside the White Cube. The Ideology of the Gallery Space, Santa Monica/San Francisco, Lapis Press, 1986, 113 p. ; Nick Kaye, Multi-media. Video - installation - performance, New York, Routledge, 2007, 272 p. 


\title{
MINIATURES: MOTIVATION ESTHÉTIQUE DE « L'OBJET TROUVÉ»
}

Il y a un certain temps que la plupart des écrivains ont abandonné l'idée d'un lexique intrinsèquement poétique. Tout mot, toute expression, sciemment utilisés, peuvent en effet être investis de littérarité, celle-ci n'étant pas fondée que sur la musicalité, la signification et l'histoire des vocables, mais également sur la connotation particulière qu'on en propose, laquelle est étroitement dépendante du contexte interne et externe du livre. Cette conscience de la «motivation » du signe va de pair avec une ouverture quasi complète quant aux thèmes potentiels de la poésie. Comme l'ont bien montré Francis Ponge, puis les tenants d'une poétique du quotidien, l'objet le plus prosaïque est susceptible de donner lieu à un poème grâce à une énonciation souveraine affirmant le rôle central de la subjectivité dans le processus de création de valeur.

Cette extension maximale du "poétisable " est naturellement compatible avec une inclusion du hasard parmi les outils de composition, ne serait-ce que comme point de départ. Une telle perspective est à l'origine d'un des plus beaux livres d'Herménégilde Chiasson, Miniatures, paru en 1995. Installation textuelle à partir d'objets divers, ce livre ne se laisse pas classer facilement, comme en témoigne le texte disposé en quatrième de couverture:

\begin{abstract}
Miniatures est un livre inspiré d'une série d'objets. De ces objets que j'avais épinglés sur un mur de bois dans une maison que j'ai autrefois habitée. Le temps est passé. J'ai remisé les objets dans une boîte que j'ai ouverte par hasard, plusieurs années plus tard. Je me suis alors rendu compte que ces objets étaient des générateurs. C'est avec ces matériaux que j'ai choisi d'écrire ces soixante textes poétiques, qui constituent une sorte d'essai autobiographique.
\end{abstract}

Parce qu'ils sont le fruit d'une sélection arbitraire, ces objets redécouverts aléatoirement ont donc servi de contrainte à un travail d'écriture où le sujet s'est projeté en verbalisant son passé. La réunion fortuite d'éléments, qui s'accompagne d'une motivation sémiotique complexe, conserve cependant une partie de son ambiguité grâce à la structure fragmentée du recueil, équivalent textuel d'une exposition où le lecteur doit lui-même parachever la liaison des soixante tableaux de prose brève. Cette participation est encouragée par l'éclectisme de ces objets (re)trouvés (papillon synthétique, boucle d'oreille, sachet de thé, photographies, photocopies, carte professionnelle, billet de banque, certificat de naissance, etc.), de même que par l'absence de subdivisions du livre, dont les seuls marqueurs métalinguistiques évidents sont le titre, la mention générique («essai autobiographique») et la quatrième de couverture².

$$
+++
$$

2 L'illustration de la première de couverture est également annonciatrice de la stratégie énonciative. Euvre de Chiasson lui-même, l'image est composée de trois rectangles superposés représentant des réalités complémentaires - femme vue de dos, galets, atelier d'artiste - auxquelles s'ajoute (au centre droit) la boucle d'oreille retrouvée à l'intérieur. Dans le mince rectangle du centre, un bâton brûlé est déposé sur les galets et porte le titre du recueil. D'entrée de jeu, nous sommes mis en situation d'assemblage et de réunion des signes. 
Petites choses, expressions métonymiques des étapes d'une vie, les soixante artefacts sont disposés sur les pages de gauche, formant un cabinet de curiosités dont les textes en pages de droite sont le commentaire et le développement. Plutôt qu'une simple valorisation du fortuit, le recueil active une tension entre l'épars et le continu, l'unité stylistique apportant un contraste qui aiguille la lecture sans fournir toutes les clés nécessaires à la cristallisation du contenu. Devant ce livre, on doit s'approprier la transition des objets à l'œuvre-livre dans une expérience du divers qui est fréquente du côté des identités américaines forgées à travers l'hybridité et le déplacement. Cette expérience est d'ailleurs lisible chez le personnage féminin décrit dans l'incipit:

\begin{abstract}
Elle arpentait les lieux de ses beaux yeux verts, sournois au-dessus de son épaule, pour voir s'il n'entrerait pas par hasard quelqu'un qu'ils avaient connu autrefois heureux et qui les surprendrait ensemble. Les sentiments qui les avaient bouleversés sinon unis s'étaient estompés depuis en un faisceau d'arabesques éclatant désormais en larges fissures noires et squelettiques. $(M, 7)$
\end{abstract}

Faut-il associer ce faisceau éclaté en fissures noires à l'artefact cubain représenté en page de gauche, à un état psychologique, ou encore à la transformation de ces deux dimensions par ces lettres que nous lisons? On sent d'emblée qu'il faudra naviguer d'un plan à l'autre, œuvrer et dés-œuvrer pour qu'une signification plus globale émane de ces juxtapositions: «Désœuvrement. Elle laissait fondre le mot dans sa bouche", raconte le locuteur, avant de relater l'instant où l'homme faisant face au personnage féminin dépose «l'objet» dans sa poche, le texte s'achevant sur un écho au perceptivisme de Berkeley et à sa maxime esse est percipi : "Il ferma les yeux et le monde disparut. Momentanément.» $(M, 7)$

Ce «il», récurrent, s'avérera le moyen terme par lequel l'auteur procédera à son autobiographie, replongeant à chaque texte dans les circonstances de cueillette d'un artefact, tout en l'adaptant à l'installation en cours. Immergé dans une pure description d'états intérieurs et de perceptions, le lecteur a cependant toujours le dernier mot, puisqu'il doit à chaque page tirer ses conclusions, imaginer l'espace considérable qu'aménagent les ellipses entre les textes et les écarts spatiotemporels abrités par les objets. Sans compter que le tracé irrégulier de ce regard sur soi, parsemé de plusieurs digressions à travers les vies des autres, empêche un rabattement univoque des divers épisodes sur le je autobiographique. Au contraire, c'est souvent la vibration chaotique bousculant le vécu qui est mise en évidence, tout comme l'échec dynamique de la mémoire:

Une nouvelle vie à tous les jours. Un slogan sournois dans la rumeur de toutes les autoroutes. Tous les jours refaire l'estimation des dégâts de la veille, se convaincre qu'il y a encore à redire sur ce qui la veille n'était encore qu'un faible indice de la racine du mal. Une précision disséminée dans des milliers de destins, dans d'innombrables versions. $(M, 21)$

À la recherche de cette "précision disséminée», Chiasson confère aux détails une souveraineté absolue, car c'est en eux que subsiste une totalité insaisissable sinon 
dans la transformation constante, dans un va-et-vient sans conclusion quant à la question du dévoilement: «Il faut déshabiller jusqu'aux souvenirs. Montrer les battements du cœur inquiet.» $(M, 119)$ Bien que « [l]'écriture ne fonctionne plus [et que] l'alphabet [ne soit] plus qu'une sculpture à la précision fondante» $(M, 125)$, il semble que l'incertitude pleine de connivence qui habite Miniatures soit à même de rendre à nouveau possible la présence, ce pourquoi le hasard et l'altérité doivent d'abord être accueillis, (re)cueillis, dans une parole qui n'aime les frontières - et d'abord celle entre l'émetteur et le récepteur - que mouvantes. Aussi l'objet retrouvé, plutôt que d'être déposé dans un catalogue muséal, amène-t-il vers une structure conversationnelle où son statut objectif est sans cesse contesté à la manière d'une installation en acte.

\section{CANALISER LE FORTUIT : CONVERSATIONS, ACTIONS, BEATITUDES}

Cette vision dialogique du réel et de l'objet se trouve radicalisée dans le livre Conversations fabriqué à même une rumeur orale que le poète découpe et infléchit vers l'écrit. Voulant réconcilier l'oralité acadienne avec l'écriture moderne, Chiasson base sa composition sur une sélection de mille fragments de conversations entendues, qu'il refond afin de tenir compte «des intervenants et d'une vision poétique qui dépasse largement le constat quotidien que l'on tient dans un journal» $\left(\mathrm{Co}, 4^{\mathrm{e}}\right.$ de couverture). Attribués successivement à $I l$ ou à Elle, ces centaines de fragments exigent du récepteur un effort constant afin qu'il leur imagine un contexte commun, dont le lieu ultime demeure celui du livre et de sa mise en lecture. Afin de donner une idée de la discontinuité doublée d'une dynamique associative qui se met en place, voici quelques répliques de ce thêâtre poétique ${ }^{3}$ :

57. Elle Comment détourner le flot de la conversation vers l'océan avoué de son ambition?

58. Elle Depuis cette époque, dans sa tête, le bruit assourdissant d'une discothèque.

59. Elle Les choses qu'on sait maladroites et dont on s'accommode, et qu'on embellit à la longue.

60. Lui Toute la patience du monde pour produire un objet à jeter aux poubelles.

61. Lui D'avoir perdu pour toujours quelqu'un qui nous tenait la main dans un monde sans merci. $(\mathrm{Co}, 14)$

Propositions disjointes issues de locuteurs divers, ces lignes appellent à lire entre elles, à leur surajouter une syntaxe seconde, laquelle mettra en jeu autant la

$$
++
$$

3 C'est Pierre Nepveu qui, dans sa préface à la réédition de Conversations (Sudbury, Prise de parole, coll. «Bibliothèque canadienne-française», 2006, 154 p.), convoque la notion de «théâtre invisible» pour désigner la polyphonie du recueil. Le statut des instances énonciatives demeurant indéfini, tout comme la dimension spatiotemporelle, la thêâtralité du discours s'avère légèrement plus métaphorique que son caractère installatif. 
relation entre hommes et femmes qu'entre humains contemporains du lecteur. Constellation de conversations fragmentaires, l'ensemble nécessite donc de l'interprète qu'il converse lui aussi, qu'il ose des associations, faute de quoi le livre risque de demeurer un inventaire inerte. Livre miroir, pourrait-on dire, combinant le thème des relations humaines avec une esthétique fortement relationnelle, où le matériau textuel, le travail de l'auteur et celui du lecteur deviennent indissociables. «Détourner le flot de la conversation " est en effet un acte partagé, réitérable, d'où ce sentiment de prolifération qui nous habite à la lecture de cet ouvrage, que nous pouvons aisément parcourir au hasard sans que son principe de composition en soit altéré.

Activité éphémère, la conversation se voit ici haussée au rang d'objet esthétique, alors que le poète ne laisse apparaître son propre art que discrètement, comme s'il se contentait d'aiguiller le train chaotique du discours quotidien, malgré une unité de ton qui laisse peu de doutes à l'égard de la reformulation des paroles empruntées. À maintes reprises, le recueil trouvera dans ces répliques une (auto) référence à son processus même, tendu entre accueil et volonté, entre l'inscription subjective et quelque rémanence d'un magma impersonnel:

\footnotetext{
806. Lui Le doute insistant de la présence, hésitant entre la matière et l'éveil, tentant démesurément de refaire le parcours dont il dessinait à la longue les cartes et les cours d'eau.

807. Elle Elle n'avait pas de modèle précis, s'accrochait aux détails de la même histoire, incapable d'inventer le moyen de se redonner une direction et, en définitive, un ensemble de mots plus ou moins conséquents. (Co, 120)
}

Structurée par une manière d'assembler les fragments, cette suite bâtit sa direction comme le ferait un improvisateur conséquent, adaptant quelques principes à l'événement collectif en cours avec une conscience du moment qui convoque tous les sens du mot concert : action de concerter ou de se concerter, collaboration musicale, atteinte de l'unisson, élévation d'une clameur.

Une poétique assez ressemblante est lisible dans le recueil Actions (2000), où l'auteur juxtaposera des centaines de fragments transposant chacun un geste observé. Encore une fois, la stratégie rappelle le ready-made, ces actions quotidiennes étant simplement énumérées sous forme de liste et leur assemblage sous forme de livre venant leur conférer une plus-value esthétique. Sous des allures de rassemblement fortuit, l'inventaire engage un regard global grâce auquel les parties dépassent leur banalité d'origine, tout en permettant un parcours de lecture discontinu qui n'affectera pas l'intégrité de l'œuvre. On peut en effet déambuler à rebours ou dans le désordre parmi ces fragments non numérotés, l'essentiel étant de les faire se rencontrer, de conférer un ordre à ce foisonnement décentré:

Une femme renverse accidentellement le vin qu'on vient de lui servir dans un verre en papier trop léger.

Un homme, avant d'aller se coucher, commande un repas qu'il mange au bord d'une piscine. 
Un homme, tombant de fatigue, écrit dans son lit la conférence qu'il doit donner le lendemain.

Un homme remet soigneusement en ordre les diverses sections du journal qu'il vient de lire. $(A, 118)$

Contrairement au journal évoqué, Actions est dénué de sections; seules quelques photographies de Raymonde April interrompent le flux des gestes recensés. L'effet d'inachèvement qui en résulte laisse place à un espace relationnel intense, qui sera notamment réitéré dans Répertoire puis dans Béatitudes, autre titre pluriel où l'énumération joue un rôle clé. Dans ce dernier cas, le recueil s'articule autour d'une série de références à des entités collectives, soit masculines soit féminines, dont les gestes et les attitudes dessinent une communauté fourmillante et dont l'identité s'acquiert à travers une mobilité baroque. Trois citations et six épigraphes précèdent le texte, entièrement constitué de désignations. L'indication et l'indexation vont alors de pair, dans une forme qui intègre à la fois l'inventaire et la scansion religieuse, ce qui s'accompagne d'un leitmotiv centré sur la notion de ciel:

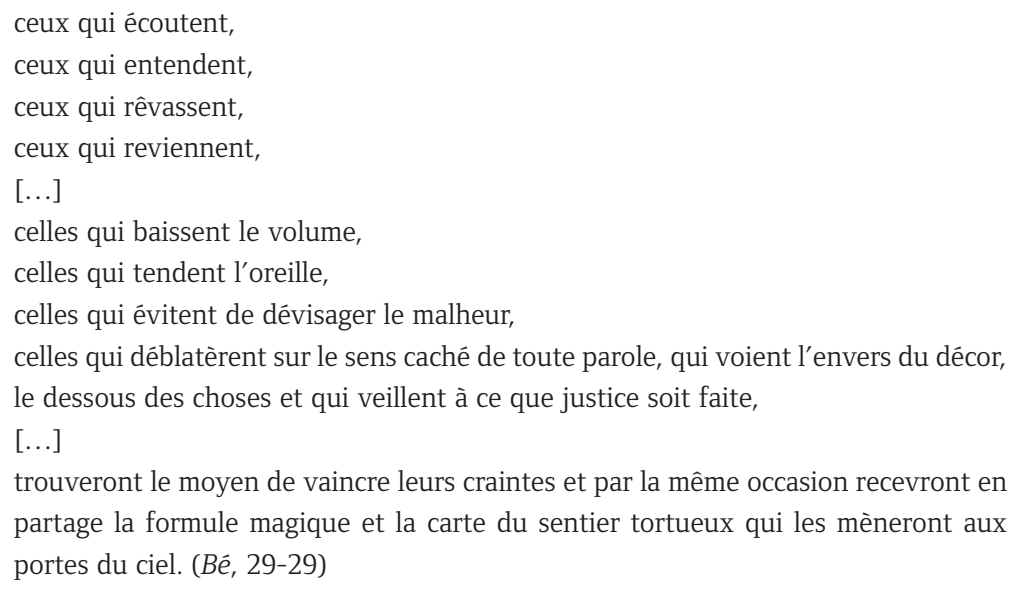

Exercice de compassion, de "passion-avec», Béatitudes est un hymne à la collectivité en soi, à ce que toute collectivité comporte d'arbitraire et de profus. C'est pourquoi le lieu où évoluent les gens est laissé indéfini, le lecteur devant lui-même imaginer un contexte concret, donner une figure aux Ceux et Celles qui se croisent sur les pages. Constitué d'une seule longue phrase, le recueil se termine d'ailleurs sur une virgule, soulignant l'infinitude du processus amorcé. Cette ouverture finale rappelle, aussi, celle du millième fragment de Conversations, laissé blanc.

\section{BRUNANTE : POÉTIQUE DE L'INTERSECTION}

L'installation autobiographique de Miniatures se prolonge sous une autre forme dans Brunante, publié en 2000 et portant la mention générique « récits ». Les courts textes 
réunis ici abordent plus explicitement des moments clés de la vie de l'auteur témoignant d'une interrogation sur la création qui s'étend de l'enfance jusqu'à la maturité, en passant par les années de formation. L'esthétique installative est encore une fois présente dans la construction de l'ouvrage - notamment par le recours aux ellipses et au collage d'événements et de références - , alors qu'on peut déceler un certain métadiscours à son propos grâce à l'omniprésence de l'art contemporain.

Dans «La rétrospective Marcel Duchamp», par exemple, l'auteur raconte son périple en compagnie de quelques amis vers de prestigieuses galeries new-yorkaises. Après avoir traversé plusieurs états américains, les compagnons trouvent un miroir ambigu de leur errance une fois placés devant les œuvres impressionnistes, abstraites ou surréalistes, mais surtout dans celles qui remettent en question le statut de l'art. Sacrant Marcel Duchamp comme «un des trois artistes majeurs» de son siècle, le narrateur décrit la fascination exercée par ses productions:

L'inframince comme source. Des cultures de poussière sur une vitre, des uniformes en fil de plomb, de l'air de Paris en bouteille, des dessins à faire tourner sur des tourne-disques, une tonsure en étoile, des objets élevés par la signature au rang d'œuvres d'art, des parties d'échecs nulles, des personnages éclaboussés dans des escaliers. Des œuvres séminales, génératrices, énigmes marquantes et provocantes de fascination. $(B r, 72)$

«Génératrice», la pratique de Duchamp l'est effectivement à l'égard de ce texte, où les anecdotes du voyage occupent beaucoup plus de place que l'objet supposé par le titre, lequel est presque relégué au statut de détail. Si la référence à Duchamp conserve son rôle central, c'est en fait parce que Chiasson l'immerge dans le tissu connotatif de la vie et en applique les présupposés en demandant à son lecteur de quitter le rôle du spectateur et de faire intervenir sa propre compréhension du vingtième siècle.

\section{PUISSANCE IDENTITAIRE DE L'IMPLICITE}

La conception baroque et polyphonique du rassemblement textuel que transmet Chiasson, on en perçoit aisément l'actualité en ces années de mondialisation, où les identités collectives tremblent, confrontées qu'elles sont à l'accélération des échanges sémantiques de tous types. Dans ces conditions, un texte peut-il durer autrement qu'en se faisant le relai d'une étourdissante mobilité, en prenant acte du bouillonnement culturel à partir duquel il tente de s'établir? En déportant une partie de la cohérence sur le terrain de la lecture et en laissant les références spatiotemporelles grandement ouvertes, le poète n'abandonne pas les siens, mais leur offre plutôt ce risque insigne consistant à jouer le jeu d'une représentation inachevée, mutante, d'autant plus forte que les autres pourront s'y reconnaître. Aussi ouvertes soient-elles, les installations textuelles de l'Acadien nous parviennent d'un lieu et d'un topos précis, soit non seulement un espace de résidence, mais aussi un dialogue commun sur la nature de la nation acadienne. Sans folklore ni thématisation 
évidente, Chiasson opte pour une affirmation par la conversation, ses livres étant appelés à modifier son être une fois lus par d'autres. Comment demeurer soi-même en se confrontant ainsi à la pluralité vertigineuse des relations humaines et des paroles. C'est là un des secrets les mieux entretenus par les recueils du poète, qui parvient manifestement à être bien de chez lui, alors même qu'il est en perpétuelle transformation.

La disparition presque complète de la référence explicite à l'Acadie est peutêtre ainsi plus activement acadienne, en ce qu'elle fait place à une stimulation identitaire incompatible avec une contextualisation trop précise. Dès les textes de jeunesse de Chiasson, Raoul Boudreau remarque d'ailleurs à juste titre la poursuite d'un universel singulier qui fournira une tension durable:

L'Acadie est bien sûr omniprésente dans ces textes, mais les sentiments de perte, d'immatérialité, d'irréalité du monde, d'impuissance sont si bien rendus qu'ils dépassent les particularismes de l'exemple qui les fait naître pour atteindre à l'universalité de l'essence qui les rend reconnaissables par le plus grand nombre. C'est le premier paradoxe de l'écriture, à savoir qu'en renonçant à présenter son expérience comme une essence on arrive à communiquer l'essence de l'expérience générale à laquelle elle se rattache ${ }^{4}$.

Paradoxalement, une dimension éthique et politique émerge de la pratique littéraire installative. En forçant son lecteur à converser, Herménégilde Chiasson lui propose de se faire lui-même cueilleur et assembleur d'objets; en refusant une geste épique explicite, en ouvrant la problématique communautaire à une possibilité autant acadienne qu'étrangère, il offre à l'identité acadienne ce pivot entre soi et l'autre qui forge les identités contemporaines. Curieux mouvement, par lequel l'intérêt pour les matériaux les plus éphémères attise le désir de durer.

4 Raoul Boudreau, préface à Émergences, Ottawa, L'interligne, coll. «Bibliothèque canadienne-française», 2003, p. 9. 